\title{
What do AP physics courses teach and the AP physics exam measure?
}

\author{
Eric W. Burkholder* \\ Department of Physics, Stanford University, Stanford, California 94305, USA \\ Carl E. Wieman \\ Department of Physics and Graduate School of Education, Stanford University, \\ Stanford, California 94305, USA
}

(Received 4 March 2019; published 26 August 2019)

\begin{abstract}
We examined the variation in Force and Motion Conceptual Evaluation (FMCE) preclass scores according to the type of physics class a student had in high school. This was done for students who had taken Physics 1 at a highly selective university. The majority of students had taken an Advanced Placement (AP) physics course, allowing us to examine in detail the correlation between FMCE score and both taking an AP course and the score on the AP exam. We also carried out regression analyses to determine how FMCE prescores and course final exam scores depend on taking an AP course and AP exam scores, when math SAT scores are included as a proxy of students' general level of college preparation. Students who take any type of physics course in high school outscore those with no prior physics experience by $26 \%$, but students who have taken AP physics do not significantly outperform those who have taken a normal high school physics course - many students who took AP physics scored very low on the FMCE. Furthermore, while taking AP physics predicts a statistically significant increase in FMCE scores, the effect size (0.30) is smaller than that seen for math SAT scores (0.47). We found that AP exam scores were not correlated with final exam scores after controlling for math SAT score. In all, the data indicate that, though taking an AP physics course is correlated with greater conceptual understanding of physics, scoring well on the AP physics exam may be a rather weak measure of conceptual understanding of physics or the mastery of physics one would expect students to achieve from an introductory university physics course. This work was done with an unusual population. Further studies are needed to determine how general the weak relationship is between AP exam performance and FMCE performance that we observed.
\end{abstract}

DOI: 10.1103/PhysRevPhysEducRes.15.020117

\section{INTRODUCTION}

Each year, nearly 300000 high school students take advanced placement (AP) exams in physics [1]. The College Board provides both algebra-based (AP Physics 1 and 2) and calculus-based (AP Physics C) versions of these examinations, and many colleges and universities allow students to place out of introductory physics courses if they receive a score of 3 or better (on a 5-point scale) on one or more of these exams. These exams are developed by a national committee of educators in physics and engineering at the high school and university level and are widely accepted as good measures of how much physics students have learned during the AP course. However, to

\footnotetext{
*Corresponding author. eburkhol@stanford.edu

Published by the American Physical Society under the terms of the Creative Commons Attribution 4.0 International license. Further distribution of this work must maintain attribution to the author(s) and the published article's title, journal citation, and DOI.
}

our knowledge, there is no peer-reviewed research demonstrating what aspects of physics learning the AP exam assesses - the research on AP programs focuses on the correlation between AP exam performance and general college preparation [2].

Concept inventories are widely used by instructors to gauge the amount of physics prior knowledge their students have and/or the effectiveness of their pedagogy (with a preand post-test to see how much students learned). Two of the most popular assessments for introductory mechanics are the Force Concept Inventory (FCI) and the Force and Motion Conceptual Evaluation (FMCE). These exams have been taken by thousands of students at many different universities across the world and are widely accepted measures of conceptual understanding of force and motion $[3,4]$. Scores on these assessments are most commonly used as proxies for conceptual learning of introductory physics.

We could find no study that examined the correlation between student performance on the AP physics exam and student performance on a physics concept inventory at either the high school or college level. One researcher used a practice AP exam to measure learning gains using 
modeling instruction in a single AP Physics C-Mechanics course, but did not report correlations with concept inventory scores and does not consider the possible confounding effects of other aspects of student preparation [5].

A study of gender gaps in introductory physics found that students who took a high school physics class earned better scores on the precourse FMCE assessment than students with no high school physics [6]. However, high school physics experience was not found to be a statistically significant predictor of conceptual learning (as measured by FMCE postscore) once other covariates such as attitudes toward physics and math preparation were included in the analysis [6,7]. (Other studies have corroborated the finding that mathematics preparation is a significant predictor of college physics performance [8].) Furthermore, there is some evidence that indicates that-beyond whether or not a student had high school physics - the quality of a student's high school physics class is an important factor in predicting college physics performance $[9,10]$.

Our research questions for this study are as follows. (1) Does taking an AP physics course correlate with conceptual understanding of mechanics as measured by the FMCE? (2) What is the correlation between student performance on the AP physics exam and student performance on the FMCE? (3) Is the AP exam score a good predictor of performance in an introductory college physics course? We hypothesize that there should be a correlation between AP score, conceptual understanding of physics, and course performance, and that AP score should be a good predictor of course performance in introductory physics, given that it is intended be a replacement for that course, and that there is substantial overlap in topics covered by the AP exam and topics covered in the introductory course of interest at this university [11]. Indeed, the AP Physics C-Mechanics exam is supposed to be "equivalent" to courses in the introductory physics sequence. We note that we can only provide limited answers to these questions, as we have data from a small and unique student population (as described below).

\section{METHODS}

To answer these questions, we analyzed the performance of 430 students whose high school physics background we knew and who were taking the introductory calculus-based mechanics course at a highly selective university during the winter quarter of 2018. This was a standard Physics 1 course, covering basic mechanics, rotational motion and angular momentum, and energy. This is nearly identical to the set of topics covered by the AP Physics C-Mechanics exam (kinematics, Newton's laws, energy, momentum, rotational motion, and oscillations) [11]. The university course adhered to the treatment in the course textbook, the widely used University Physics, with Modern Physics by Young and Freedman [12]. The course was typical of most universities, with lectures that were largely traditional but with a few clicker questions interjected, and weekly recitation sections in which students solved problems working in small groups. Many of the problems were exercises taken from Tutorials in Introductory Physics [13]. The FMCE concept inventory was administered in the conventional manner used in physics education research (PER) and the routine monitoring of student learning common in such courses. Students were given the FMCE as a zero-stakes precourse diagnostic during the first week of the term. The FMCE was not graded and students never saw their scores; students were given class participation credit for completing it. The students completed the FMCE again during their recitation section in the final week of the course. The postcourse FMCE was similarly zero stakes in that it was not graded and students were not given feedback on their performance. They were encouraged to complete it for participation credit and to help guide their study for the final exam.

The physics background of the class was self-reported as follows: 45 students had taken no prior physics courses, 59 had taken high-school physics, 192 had taken an AP physics course, 104 had taken a non-AP algebra-based college-level physics course (the majority are likely International Baccalaureate physics students), and 30 had taken another calculus-based college physics course. Of these 430 students, 7 reported scores for the AP Physics 1 exam and 44 reported scores for the AP Physics CMechanics exam; the average score on both exams was 4 (s.d. =1). There are limitations when working with selfreports (e.g., students who felt they performed poorly might underreport their prior physics experience). However, students reported this experience at the end of the FMCE pretest, so we expect the zero-stakes nature of the assessment and the fact this was at the start of the course gave little reason for the students to answer incorrectly.

AP exam scores were received from the office of institutional research. Of these 50 students who reported scores for the AP exam, 48 reported taking an AP physics course, while two reported taking only high school level physics. The average math SAT score (also received from the office of institutional research) among all students was 730 (s.d. = 50) [14]. The average score on the FMCE, which was administered as a diagnostic assessment at the beginning of the course, was 53\% (s.d. $=27 \%$ ); the average FMCE score when administered in the last week of class was $77 \%($ s.d. $=26 \%$ ) [16]. These SAT scores and FMCE prescores are higher than most universities; this is a somewhat unusual student population. However, the high prevalence of students that have taken an AP physics course and the large spread in FMCE scores (Fig. 1) help us to address the research questions.

(1) In determining whether AP physics experience and/ or outcomes correlate with students' conceptual understanding of mechanics, we perform several analyses, which we describe briefly below, and in 

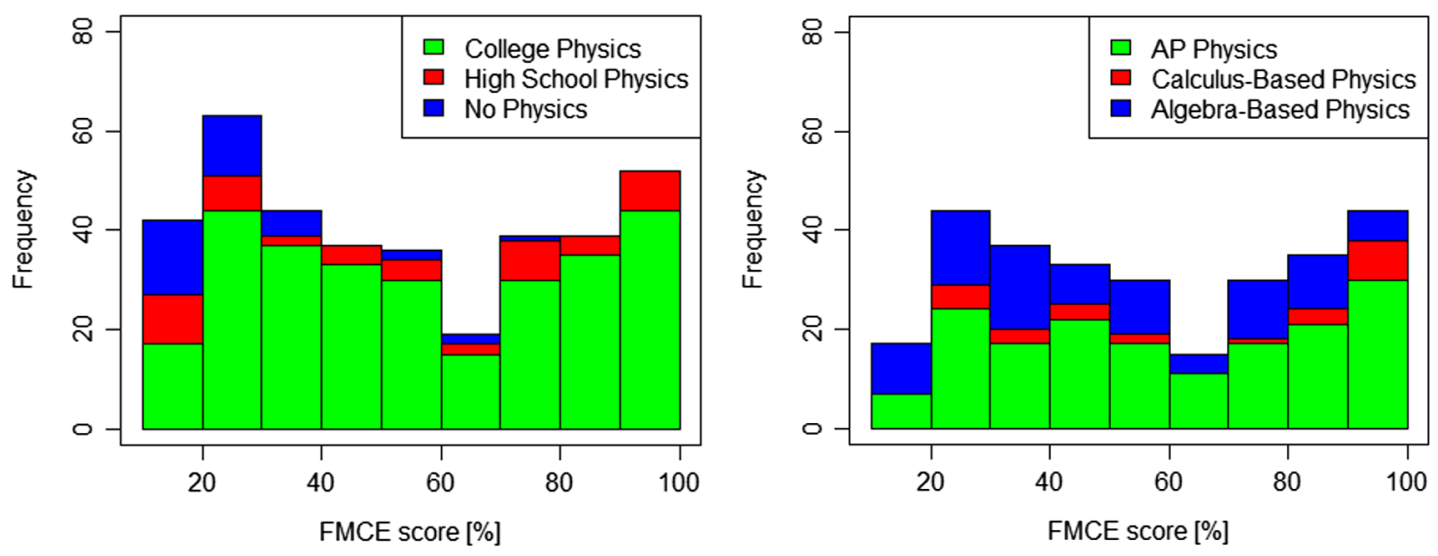

FIG. 1. Left: Histogram of FMCE prescores for students with no prior physics background (blue, $N=45$ ), high school physics (red, $N=57$ ), and AP or college physics (green, $N=328$ ). Right: Histogram of FMCE prescores for students with college-level physics.

greater detail in the Appendix. These analyses are complicated by the fact that all of the datasets have non-normal distributions, including having a variety of awkward shapes and asymmetries. This requires the use of more complicated ("nonparametric") statistics that, unlike the more familiar t-tests and analysis of variance (ANOVA), are insensitive to the non-normal shapes of the distributions. We look at the distribution of FMCE prescores by type of prior physics preparation: AP physics, calculus-based college physics, algebra-based college physics, high school level physics, and no physics.

(2) We test for specific between-group contrasts to determine the difference in prescores between students who take AP physics and those who do not. Because the data are not normally distributed, we employ the nonparametric Kruskal-Wallis test. This test is used for comparing multiple samples when the data are non-normally distributed using the withingroup ranks and sample sizes; the distribution of the test statistic follows a chi-squared distribution with $g-1$ degrees of freedom, where $g$ is the number of groups being compared [17]. To look at pairwise comparisons, we computed the Hodges-Lehmann estimator (HLE) for the Mann-Whitney $U$ test, and corrected $p$ values for multiple comparisons using the Benjamini-Hochberg procedure [18]. The HLE is a quantity that compares the difference between two distributions that is insensitive to the shapes of the distributions. It finds the median of the pairwise differences between all points in the two distributions. It is an effect size that is not scaled by the sample standard deviation [19].

(3) We compare these students' FMCE prescores with their math SAT or ACT score (using the same methods as in the prescore comparison). As we had a mixture of both SAT and ACT scores, we converted the ACT scores into SAT scores using the concordance table [14]. For simplicity, these are just labeled as "SAT" in the remainder of the paper. As the FMCE involves no math, we interpret the math SAT score as a proxy for their general level of college science, technology, engineering, and mathematics (STEM) preparation. In other work [20] we have seen that math SAT scores significantly correlate with a variety of physics specific outcomes, while other SAT scores do not.

(4) We use multiple linear regression to simultaneously examine the relative contributions of how both taking AP physics (or a college-level physics class) and math SAT score contribute to a student's predicted performance on the FMCE. We converted FMCE and SAT scores to $z$ scores so that the regression coefficients may be interpreted as effect sizes. To evaluate the regression models, we looked at the statistical significance of the regression coefficients, as well as the adjusted R-squared value for the regression, which is equal to the proportion of variance in FMCE prescores explained by the various predictors (with a penalty for increasing model complexity [21]). We use ANOVA to test for significance of the differences between nested regression models; models are nested if a simpler model contains a subset of the variables included in a more complex model [21].

To answer the second research question, we repeat the multiple linear regression on the subset of students who reported AP physics exam scores, examining how both AP score and SAT score are correlated with FMCE prescore. To gain more insight into potential mechanisms for these correlations, we then decompose the FMCE into three factors identified by previous work [22]. (We do not perform a complete factor analysis, as that is beyond the scope of this paper.) We look at correlations of these factor subscores with AP exam score and SAT score to determine if there are specific content areas for which taking AP physics has a larger impact. To answer the third research question, we 
TABLE I. Pairwise comparisons of FMCE prescores by prior physics experience. Differences between pseudomedians are computed using the Hodges-Lehmann estimator (HLE), and the statistical significance of each difference is computed using the Mann-Whitney $U$ test, corrected for multiple comparisons. The sign of HLE estimates is determined by column group minus the row group. $* p<0.05, * * p<0.01, * * * p<0.001$.

\begin{tabular}{lccccc}
\hline \hline & \multicolumn{1}{c}{ None } & AP physics & $\begin{array}{c}\text { Calculus-based } \\
\text { college }\end{array}$ & $\begin{array}{c}\text { Algebra-based } \\
\text { college }\end{array}$ & $\begin{array}{c}\text { High school } \\
\text { physics }\end{array}$ \\
\hline AP physics & HLE $=-28 * * *$ & & & & \\
Calculus-based college & HLE $=-28 * * *$ & HLE $=-2.1$ & & & \\
Algebra-based college & HLE $=-19 * * *$ & HLE $=6.4$ & HLE $=8.5$ & & \\
High school physics & HLE $=-23 * * *$ & HLE $=5.3$ & HLE $=6.4$ & HLE $=-2.1$ & HLE $=-8.5$ \\
AP test & HLE $=-34 * * *$ & HLE $=-6.4$ & HLE $=-2.1$ & HLE $=-13 *$ & HLE \\
\hline \hline
\end{tabular}

compute the Pearson product-moment correlation between AP exam score and course final exam score for the same subset of students, as well as the partial correlation between AP exam score and final exam score when controlling for math SAT score.

\section{RESULTS}

Figure 1 shows the distribution of FMCE scores for students with various levels of physics course in high school. There is a clear floor effect for students with no prior physics experience: nearly three-quarters of these students receive a score within one standard deviation of random guessing (which adds up to $15 \%$ ). For students with high school physics background, there is wide variation-some students perform quite poorly, but an almost equal number score quite well (greater than $85 \%$ ). There is also a very wide distribution of scores for people who had taken a college-level physics course: nearly $10 \%$ of these students scored below 20\%. The distribution of scores for students who took college-level physics does not vary substantially between groups.

We first used the Kruskal-Wallis test, an analysis of variance appropriate for nonparametric distributions, to test for the significance of the overall variations in score by the five different types of preparation. We found that FMCE prescores did vary significantly by reported prior physics experience $\left[\chi^{2}(5)=60.2, p<0.0001\right]$. We then carried out a pairwise comparison (Table I). This shows that students with any prior physics experience outperform those with no prior experience-the pseudomedian is between 19 and 34 percentage points higher (column 1) depending on the type of course and is 26 percentage points higher in aggregate. This is consistent with the findings of Kost et al. [6,7]. There is a statistically significant difference between the scores of students who take the AP exam and scores of those who took an algebra-based college physics course; otherwise, all forms of prior physics seem to provide an equal advantage on the FMCE pretest relative to no high school physics. Students who take AP physics do not uniformly outperform students who take other types of physics courses in high school.
We expect that prior preparation in physics is also correlated with more general measures of college preparation, for which we use math SAT score as a proxy. Figure 2 shows the distribution of SAT math scores for the various groups of students. The difference here is not obvious visually, as there is a ceiling effect on the math scores. However, a Kruskal-Wallis test confirms that the math scores do vary by prior physics experience $\left[\chi^{2}(5)=12.3\right.$, $p=0.031]$. A pairwise comparison (again corrected for multiple comparisons) shows that the only statistically significant pairwise difference is between students who report AP exam scores and students with no prior physics $(\mathrm{HLE}=37, p=0.01)$.

To look at the relationship between FMCE score, prior physics experience, and math SAT score, we use multiple regression analysis using a combination of continuous and logistical variables. In Table II we summarize the results of five different models: (1) FMCE prescore as a function of whether or not a student took AP physics, (2) FMCE prescore as a function of whether or not a student took any college-level physics, (3) FMCE prescore as a function of SAT math score, (4) the combination of models 1 and 3, and (5) the combination of models 2 and 3.

Students who take AP physics on average score 0.38 standard deviations higher (10 percentage points) on the FMCE than their peers with high school and other prior

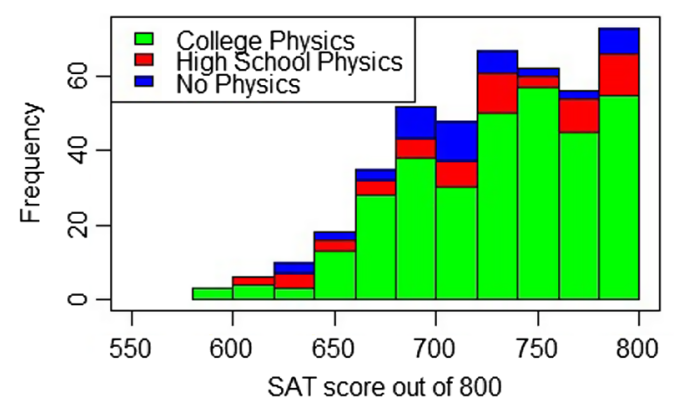

FIG. 2. Histogram of SAT math scores for students with no prior physics background (blue, $N=45$ ), high school physics (red, $N=57$ ), and AP or college physics (green, $N=328$ ). Scores are combination of reported SAT scores and ACT scores converted to SAT scores using concordance tables. 
TABLE II. Regression of FMCE prescore on various measures of prior preparation. Each variable is normalized such that the regression coefficients may be interpreted as effect sizes (Cohen's $d$ ). The standard error for each coefficient is given in parentheses. Goodness of fit of the model is determined by adjusted $\mathrm{R}^{2} . * p<0.05, * * p<0.01, * * * p<0.001$.

\begin{tabular}{|c|c|c|c|c|c|}
\hline & Model 1 & Model 2 & Model 3 & Model 4 & Model 5 \\
\hline Intercept & $-0.17 * * *(0.064)$ & $-0.39 * * *(0.096)$ & $2.8 \times 10^{-16}(0.042)$ & $-0.13 *(0.056)$ & $-0.31 * * *(0.085)$ \\
\hline AP physics & $0.38 * * *(0.095)$ & & & $0.30 * * *(0.084)$ & \\
\hline College physics & & $0.51 * * *(0.11)$ & & & $0.40 * * *(0.098)$ \\
\hline SAT & & & $0.48 * * *(0.042)$ & $0.47 * * *(0.042)$ & $0.46 * * *(0.042)$ \\
\hline Adjusted $\mathrm{R}^{2}(\%)$ & 3.3 & 4.6 & 23 & 25 & 26 \\
\hline
\end{tabular}

physics courses, or no prior physics; this explains 3.3\% of the variation in scores (model 1). Students who take any college-level physics course (including AP physics) in high school perform significantly better $(0.51$ standard deviations, or 14 percentage points) than their peers with no prior physics or regular high school physics; this explains $4.6 \%$ of the variation in FMCE prescores (model 2). Model 3 shows that there is also a statistically significant correlation between math SAT scores and FMCE prescores: for every standard deviation (50-point) increase in SAT scores, students on average score 0.48 standard deviations (13 percentage points) higher on the FMCE. When looking at the model combining SAT scores and AP physics (model 4), taking AP physics uniquely accounts for only $2 \%$ of the variation in FMCE prescore, but it is still a statistically significant improvement over model $3, F(1)=12.4$, $p=0.00048$, though the effect size for SAT math score (0.47 standard deviations increase per standard deviation increase in SAT score) is larger than that for taking AP physics (0.30 standard deviations). When comparing all students with college-level physics to those with high school or no prior physics (model 5), the effect size for SAT score is comparable to model 3 (0.46), and the effect size for prior physics experience is slightly reduced over model 2 to 0.40 ; prior physics experience uniquely accounts for 3\% of the variation in FMCE prescores.

To address our second research question, the relationship between AP exam score and other measures of physics

TABLE III. Regression of FMCE prescores on various measures of prior preparation only for students who took the AP test. Each variable is normalized such that the regression coefficients may be interpreted as effect sizes (Cohen's $d$ ). The standard error for each coefficient is given in parentheses. $* * * p<0.001$, $* * p<0.01, * p<0.05$.

\begin{tabular}{lccc}
\hline \hline & Model 1 & Model 2 & Model 3 \\
\hline Intercept & $-8.6 * 10^{-17}$ & $2.5 * 10^{-16}$ & $1.9 * 10^{-16}$ \\
& $(0.13)$ & $(0.13)$ & $(0.12)$ \\
AP score & $0.38^{* *}(0.13)$ & & $0.15(0.16)$ \\
SAT & & $0.48^{* * *}(0.13)$ & $0.39 *(0.16)$ \\
Adjusted & 13 & 21 & 21 \\
$\mathrm{R}^{2}(\%)$ & & & \\
\hline \hline
\end{tabular}

performance, we repeated the regression analysis for the subset of students for whom we had AP exam scores $(N=50)$. The results are given in Table III. The correlation between AP score and FMCE prescore is much stronger than simply taking an AP physics course (adjusted Rsquared $13 \%$ vs $3.3 \%$ ). For every standard deviation increase in AP exam score, the model predicts a 0.38 standard deviation increase in FMCE score (this amounts to an increase of 4.9 percentage points on the FMCE for each one-point increase in the AP exam).

However, the picture is more complex than this would suggest. SAT math score is a better predictor than the AP score of the FMCE score. A model that includes only math SAT score (model 2) is as good a predictor of FMCE score as model 3, which also includes the AP score. Both have an adjusted $\mathrm{R}^{2}$ of $0.21[F(1)=0.93, p=0.34]$. This is also reflected in the fact that the coefficient for the AP score in the model is about one-third as large as the math SAT coefficient, and with a much larger fractional uncertainty. The correlation between AP exam score and FMCE prescore overlaps entirely with the correlation between SAT math score and FMCE prescore, as illustrated in Fig. 3. We note that the correlation between SAT math score and FMCE prescore is not large enough (and the number of observations is not small enough) to raise concerns about multicollinearity. The variance inflation factor for these factors is 1.5 , meaning the standard error of the regression coefficients is inflated by roughly $50 \%$ over what it would

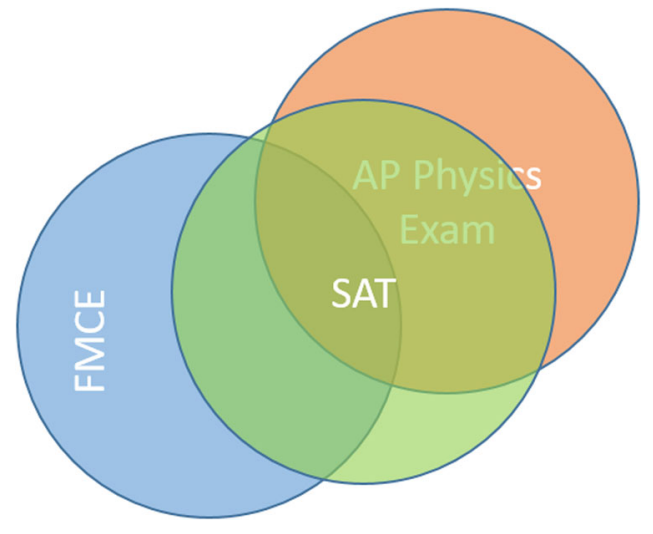

FIG. 3. Illustration of overlap between score variation on the FMCE, the math SAT, and the AP physics exam. 
TABLE IV. Product-moment correlations for AP exam score with various factors comprising the FMCE.

\begin{tabular}{lc}
\hline \hline Factor & $\begin{array}{c}\text { Product-moment } \\
\text { correlation }\end{array}$ \\
\hline Factor 1 $(1-12,14,16-19,21$, & $r=0.38, p=0.0072$ \\
25, 27, 29) & $r=0.22, p=0.12$ \\
Factor 2 $(30-32,34-36,38-39)$ & $r=0.50, p=0.00024$ \\
Factor 3 $(33,37,40-47)$ & \\
\hline \hline
\end{tabular}

be if AP score and SAT score were independent. Thus, for the coefficients in Table III, the smallest estimate of the 95\% confidence interval is $\hat{\beta} \pm 0.21$, where $\hat{\beta}$ is either regression coefficient. This does not change the qualitative result: AP score is not significant at the 0.05 level, while SAT score is.

One possible problem with this analysis is that it might be underestimating the importance of general preparation, and as a result overestimating the impact of the AP course and exam scores. Our proxy of general STEM preparation, math SAT score, is obviously quite limited. In addition to the obvious limitations about the extent of knowledge and reasoning that it measures, for this population this exam also shows a strong ceiling effect, as seen in Fig. 2, which limits its sensitivity to differences. At an institution with a broader range of math SAT scores, the additional variation would give it greater predictive power. If the FMCE distribution were more positively skewed (as one might expect when SAT scores are lower), the predictive power of SAT scores would increase even further, as the variation in FMCE scores would be reduced. All of these effects would tend to reduce the contribution of AP score to the FMCE score relative to what we report here.

We looked at performance on various portions of the FMCE to determine if there were specific concepts where students who took AP physics outperformed their peers who did not. Adopting the factor analysis from Ramlo [22], we looked at three factors. Correlations between each factor and AP score are reported in Table IV. We note that this is not a rigorous factor analysis, nor is it intended to be. This is only to provide a descriptive picture of particular topics that may be more strongly related to performance on the AP physics exam to, for example, see if there could be an obvious content mismatch between the two exams.

TABLE V. Multiple regression analysis of FMCE subscores on AP exam scores and SAT scores. $* * * p<0.001, * * p<0.01$, $* p<0.05, . p<0.1$.

\begin{tabular}{lcc}
\hline \hline & Factor 1 & Factor 3 \\
\hline Intercept & $3.9 \times 10^{-16}(0.12)$ & $8.7 \times 10^{-17}(0.12)$ \\
AP score & $0.15(0.16)$ & $0.27 .(0.15)$ \\
SAT & $0.38^{*}(0.16)$ & $0.38^{*}(0.15)$ \\
Adjusted $\mathrm{R}^{2}(\%)$ & 20 & 32 \\
\hline \hline
\end{tabular}

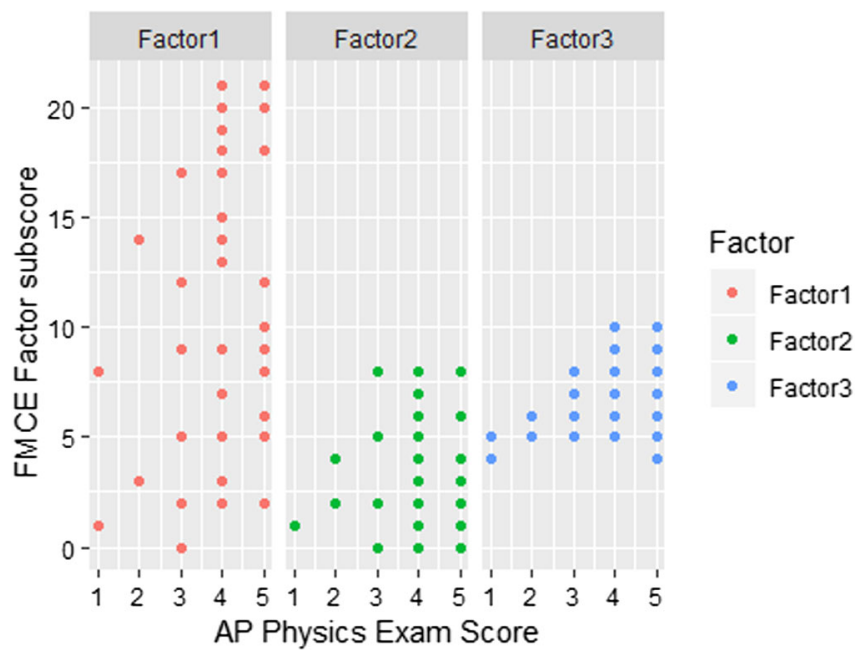

FIG. 4. Subscores on the FMCE factors for students who took the AP exam.

After correcting for multiple comparisons, AP exam score is only significantly correlated with factors 1 and 3 , which consist largely of questions on the topics of energy, graphical representations, and force or Newton's second law. A multiple regression analysis (Table V) shows that neither of these correlations is significant after controlling for SAT math score. The correlation between AP exam score and factor 2 (which probes student understanding of Newton's third law) is not statistically significant ( $p=0.12$ ). The scatter plots in Fig. 4 show that students who take the AP exam score are not approaching a ceiling on any of these sets of questions, unlike what is observed on the FMCE when administered at the end of the Physics 1 course in this and many other university cases.

Finally, we look at the relationship between AP physics and the Physics 1 final exam score. We find a small correlation between taking AP physics and final exam performance (adjusted $\mathrm{R}^{2}=0.017, p=0.0060$ ). The partial correlation is smaller when we control for math SAT score (partial $\mathrm{R}^{2}=0.011, p=0.033$ ). Among students who took the AP exam, there is a moderate, statistically significant correlation between students' performance on the course final exam and their AP score (adjusted $\mathrm{R}^{2}=0.14, p=0.0067$ ). If we control for math SAT scores, the partial correlation is no longer statistically significant (partial $\mathrm{R}^{2}=0.034, p=0.20$ ).

\section{DISCUSSION}

Our analysis first examined the relationship between type of prior physics preparation (no preparation, high school physics, and AP physics) and student performance on the FMCE at a single, highly selective institution. We found that students who had any high school physics performed better than their peers who had no prior physics class. Students who had taken an AP physics course did not significantly outperform those who had taken other physics 
courses in high school, including normal high school physics classes. Additionally, the distribution of FMCE prescores for students who had taken an AP physics course was quite wide. The FMCE is a conceptual evaluation which should be easy if students have learned the core concepts of mechanics; our data suggest that is not necessarily the case after a year of high school physics, whether or not it is AP.

When we included math SAT score as a proxy of more general measures of preparation in STEM, we found that students who take an AP physics course receive FMCE scores 0.30 standard deviations higher on average than those who do not take AP physics, while each standard-deviation increase in SAT scores predicts a 0.47 standard-deviation increase in FMCE scores. It is surprising that the measure of physics-specific preparation is a worse predictor than SAT scores in what is a physics-specific test, particularly given that AP physics is frequently accepted as a substitute for taking a university Physics 1 course. This suggests that taking an AP physics course per se may provide minimal learning of the physics concepts covered in Physics 1, and many students complete such courses with very little conceptual understanding, as shown in Fig. 1. We note that the data suggest this to be true of any college-level physics course that students reported taking in high school.

We found that the AP exam score explained no unique variation in FMCE prescores once controlling for math SAT scores. This is a striking and rather surprising result. The FMCE is entirely qualitative, so one would not expect math skills, what the math SAT is directly measuring, to be at all useful for predicting performance on the FMCE test, and certainly it should not be as good as the AP physics exam.

Upon closer examination of student performance on the FMCE by topic, we found no correlation between students' subscores on factor 2-which largely covers Newton's third law-and their AP exam score. Most instructors will find the lack of correlation on questions pertaining to Newton's third law unsurprising; students have deeply rooted misconceptions of this topic based on observations of collisions between objects. Thus, it is not surprising that many students still do not understand this concept, even after taking an AP physics course.

Closer qualitative analysis of the questions for which there was a correlation between AP exam score and topical subscore shows that the only physics-specific knowledge required to answer these questions is (1) the relationship between force, acceleration, and velocity and (2) the relationship between kinetic mechanical energy and speed, and the relationship between elevation and gravitational potential energy. A student could simply recall a few formulas and use logic to reason the answers to the remaining questions, which supports the hypothesis that the benefit of the AP exam is largely familiarity with various physics definitions, and could explain why math SAT score is a better predictor of performance on these questions. There is very little peer-reviewed psychometric information about the

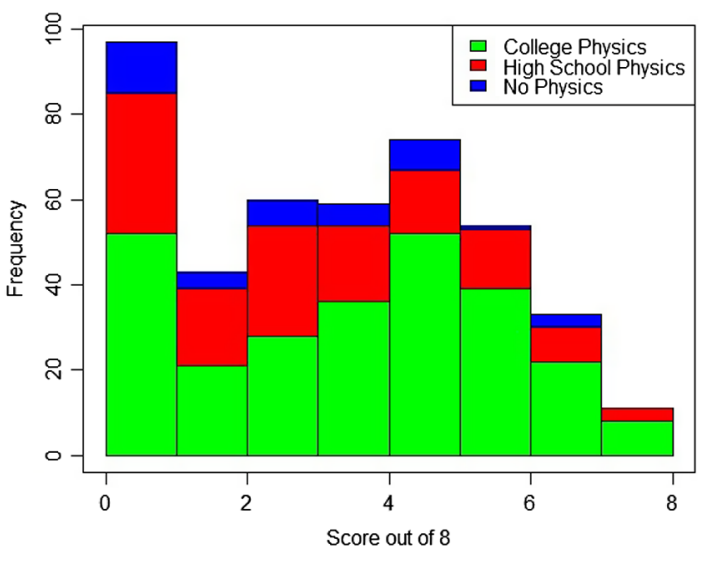

FIG. 5. Subscore on test of understanding vectors for students with various physics backgrounds. Green is students who have taken college-level physics (AP, $N=258$ ), red is for students who have taken high school physics $(N=135)$, and blue is for students who have taken no physics $(N=38)$.

math SAT; we only have access to reports of its correlation with grades in first-year mathematics courses [23]. It remains an open question as to what aspects of learning might be responsible for the correlation between FMCE scores and math SAT scores.

Another piece of evidence to support the hypothesis that the value of an AP physics course is largely familiarity with physics terms comes from an analysis conducted in the same course during the winter quarter of 2019 at the same university. We added 8 basic questions from the Test of Understanding Vectors (TUV), a research-based assessment, to the FMCE [24]. The questions covered addition and subtraction of vectors, calculating vector magnitudes, and defining the dot and cross products. The histogram of responses is shown in Fig. 5. The most common score in the class was zero- a plurality of students had no understanding of how to use vectors, and $50 \%$ of these students had taken college-level physics in high school.

Finally, we found no statistically significant correlation between AP exam score and final exam score in the courseall of the shared variance between the two is explained by math SAT scores. Given that the AP exam and the course final exam are in principle supposed to be measuring the same thing, they are nominally covering the same topics, this is surprising. This is consistent with other studies that find minimal correlations between AP exam performance and college performance outcomes (e.g., GPA) but contradicts the claim that "AP Exam scores accurately represent students' achievement in the equivalent college course" $[11,25-28]$.

\section{CONCLUSIONS}

The analysis presented in this study is quite limited in scope: we have data on a single introductory physics course for one year at a single, highly selective university. It would 
be interesting to see if similar results are obtained when looking at students with a broader range of backgrounds, particularly as reflected in their math SAT scores. It also would be worthwhile to see whether this analysis extends to introductory courses in electricity and magnetism, or even courses outside physics. An additional concern is whether our use of the AP exam score has a biased sample because of the number of students who received a 5 on AP Physics C-Mechanics and opted out of the course in question. There are 33 students who scored a 5 and took honors-level introductory physics rather than this Physics 1 course. However, there are still 12 students with scores of 5 in our sample. With those 33 students removed from our sample, our sample population is actually similar to the national distribution for AP exam scores, as well as being less sensitive to potential ceiling effects [1].

It is interesting that so few of the students who took AP physics $(25 \%)$ actually reported AP exam scores. One potential explanation is that students who felt unprepared after taking the AP course did not want to take-or were discouraged from taking - the AP exam. Another is that this university strongly disincentivizes students to take the AP exam with their strict requirements for claiming course credit [29]. There is no current data regarding this matter, as The College Board does not publish data on students who take AP courses-only those who take AP exams [30]. There have also been substantial changes over the past few years to the structure of the algebra-based physics AP exams and in participation rates in the AP program [33]. It is not the aim of this paper to speculate about the reasons for students not taking the exam, but a mixed-methods study investigating why students choose not to take the exam and their performance on practice AP tests would give some insight as to how this might affect the distribution of scores on the AP test.

This study raises questions about what exactly the AP physics test is assessing. Scores on the AP physics exam seem to be minimally correlated with conceptual understanding of physics - surely this is not what one would hope would be achieved by an AP physics program. One potential explanation for this lack of correlation is that high school students' understanding of physics is largely procedural. This is indirectly supported by previous research [34]. They may recognize when to use appropriate formulas to solve physics exercises, but without understanding the underlying physics of the problem. Indeed, this is what many college instructors discovered during the advent of the concept inventory. We note that our analysis does not show that AP courses have no impact on students' conceptual understanding of physics, but the correlation between performance on the AP exam is far smaller than we expected.

In Fig. 6 we plot the distribution of FMCE scores controlling for math SAT scores. For students who have taken college or high school physics, we plot their
Physics 1 prescores, and for students with no prior physics, we plot their Physics 1 postscores. This is effectively comparing how much physics tested by the FMCE that a student knows after their first physics course, whether it is a high school course (including AP, which is statistically indistinguishable from other high school physics courses in this sample) or an introductory university course. After correcting for differences in SAT scores, we find a statistically significant difference between the groups: post-FMCE scores of students with no prior physics are 1.4 standard deviations (21 percentage points) higher than pre-FMCE scores of students who took physics in high school, given the same math SAT scores. This indicates that a student would likely learn more physics in the introductory university course than in a high school or AP course. This comparison is confounded by the fact that the Physics 1 pre-FCME is a substantially delayed post-test for students who took high school or AP physics, while there is no delay for the students with no prior physics before taking the post-FMCE. However, Deslauriers and Wieman [35], as well as Pollock [36], found that students' retention of basic physics concepts is quite high on timescales of a year. Particularly given the large size of the effect, it seems quite likely that the relevant learning is superior in the introductory college course.

However, students with no prior physics clearly are not mastering the concepts of force and motion from Physics 1. For a visual of all students' conceptual understanding of mechanics after an introductory college course, see Fig. 7. The post-test scores for students that took physics in high school are largely at the test ceiling, while the distribution for students with no prior physics course is much lower.

Figures 6 and 7 have important implications for instruction: it appears unrealistic for instructors to expect that students with no physics background will reach close to the same level after one term of university physics as students

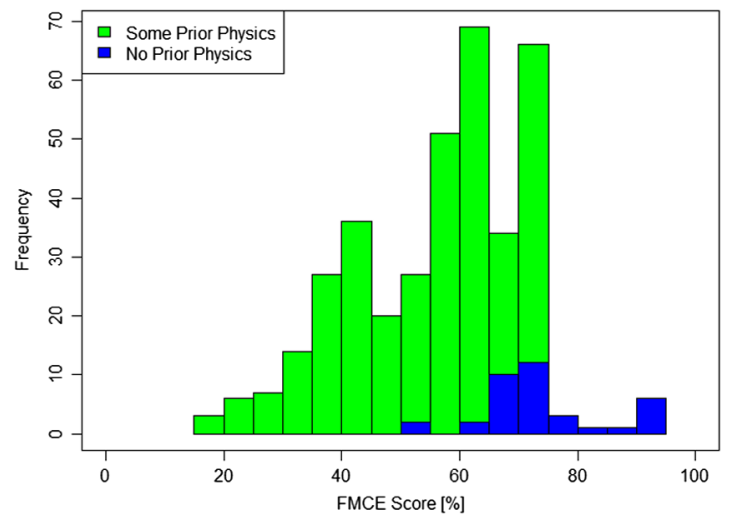

FIG. 6. FMCE score after first physics course, controlling for math SAT scores. Green bars represent the distribution of scores for students who took physics in high school $(N=337)$, and blue bars represent those students with no physics in high school $(N=37)$. 


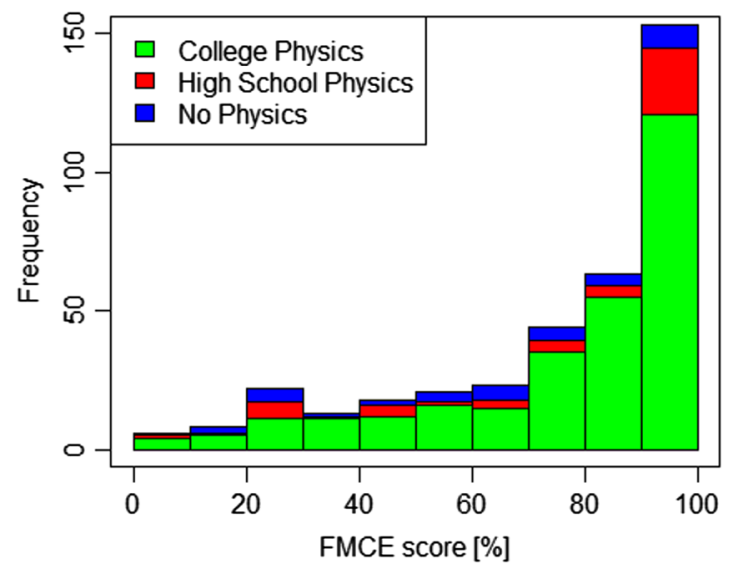

FIG. 7. FMCE postscore by prior physics preparation for students who have taken an AP or college-level physics course in high school (green, $N=286$ ), students who took a regular high school physics class (red, $N=51$ ), and students with no prior physics (blue, $N=37$ ).

who have also taken a regular high school or AP physics course 1 . Students who have taken a physics class in high school have much higher postscores. Presumably this is because of repeated exposure to the material, as these data indicate that few students master the concepts of force and motion after their first physics course. It seems plausible that the differences would be larger with student populations with lower math SAT scores than the sample we analyzed. It remains an open question for physics education researchers as to how different teaching practices might affect these outcomes and the sensitivity to students' prior preparation.

Though this study focuses in part on what the AP exam is measuring, it also raises questions about what the FMCE is measuring. The FMCE was designed based on careful qualitative research into common student misconceptions in introductory physics, and has been widely implemented, but there have been relatively few psychometric studies on the FMCE $[22,37,38]$. Recent work applying item response theory to the FCI has shown that the skills a concept inventory is actually measuring may not align well with the topics it covers [39]. We looked at the FMCE according to Ramlo's factor analysis here, but other evidence for the instrument's multidimensionality, and the suggested differential functioning in this article, highlights the need for more sophisticated psychometric analysis.

The implications for how university physics departments should view AP physics seems clear. Without further evidence, they should not assume that a high score on the AP physics exam provides any indication that a student has a solid conceptual understanding of introductory mechanics. For our sample, few students seem to have mastered the concepts of Physics 1 covered by the FMCE after their first physics course, regardless of where it was taken. The FMCE concept inventory likely provides more information about students' mastery of introductory college physics than the AP exam. As we show in Ref. [20], concept inventory prescores explain a large fraction of students' performance in physics even after controlling for general measures of incoming preparation and demographic variables [20]. Furthermore, we see that scores on the AP physics exam are too weakly correlated with students' performance on the FMCE or final exam in the Physics 1 course to provide any confidence that it is a suitable substitute for a university introductory mechanics course. Other departments should see if this is true for their students.

\section{ACKNOWLEDGMENTS}

This research was determined exempt from IRB review under Stanford University protocol IRB-48006. The authors thank Argenta Price for her input on the manuscript.

\section{APPENDIX: STATISTICAL METHODS}

This paper uses a number of statistical methods not commonly seen in PER; we therefore describe our methods in greater detail here.

\section{Nonparametric hypothesis tests and false discovery rates}

The first analysis we perform is a test to determine the statistical significance of between-group differences in FMCE prescores; this is typically done with t-tests (two groups) or ANOVA (multiple groups). Both groups test the hypothesis that the population mean of the FMCE prescore is the same in multiple groups:

$$
\mu_{1}=\mu_{2}=\cdots .
$$

These tests rely on the assumption that the sample mean of the FMCE prescore is normally distributed:

$$
\bar{x}_{1} \sim N\left(\mu_{1}, \sigma_{1}^{2}\right), \quad \bar{x}_{2} \sim N\left(\mu_{2}, \sigma_{2}^{2}\right), \ldots,
$$

where $\sigma_{i}^{2}$ is the population variance of population $i$. Under these assumptions, the test statistic,

$$
t=\frac{\bar{x}_{2}-\bar{x}_{1}}{\sqrt{\sigma_{1}^{2} / N_{1}+\sigma_{2}^{2} / N_{2}}},
$$

follows a student $t$ distribution with $N_{1}+N_{2}-2$ degrees of freedom, where $N_{i}$ is the size of population $i$. (The variance follows a $\chi^{2}$ distribution with $N_{i}-1$ degrees of freedom; the student $t$ distribution is the ratio of the normal distribution and the $\chi^{2}$ distribution.) The student $t$ distribution has known critical values, i.e., $95 \%$ of values fall within the range $[-1.96,1.96]$. These critical values establish the type 1 error (false discovery) rate we use to establish statistical significance. For example, if $t=3.1$, we know that there is only a $0.2 \%$ chance that the two 
population means are different $(p=0.002)$ only as a result of statistical fluctuations.

In the samples considered in this paper, the data are not normally distributed. The distribution of FMCE scores is nearly flat, and the distribution of SAT scores has a large negative skew; the data do not meet the criteria necessary for t-tests and ANOVA to give reliable results. This requires a way to look at group differences and calculate the range of statistical fluctuations which is valid for nonnormal distributions, which go under the general label of nonparametric tests. Instead of testing whether the means of two samples are different, nonparametric tests rank all of the data in all sets and compare differences across the entire distribution. This is more appropriate for substantially skewed distributions, as it gives a picture of global differences rather than average differences. For example, the Mann-Whitney $U$-test statistic $U$ is the number of times that each observation in one sample is larger than any other observations in the second sample (count 0.5 for a tie). The probability of observing various values of $U$ due to random statistical variations, as opposed to causal factors, can be found in textbooks on nonparametric statistics [19]. These are suitable $p$ values for hypothesis testing with nonparametric distributions.

We correct our $p$ values in Table I to account for the multiple pairwise comparisons made. The motivation behind this is straightforward and the same as when analyzing parametric distributions: a single $p$ value can be interpreted as the chance that a given result is a false positive. Typically, we choose the threshold for statistical significance to be $\alpha=0.05$. However, if we were to perform 20 independent hypothesis tests, we would expect at least one of them to be significant at the 0.05 level. A conservative correction (the familiar Bonferroni correction) is to simply multiply the $p$ value by the number of comparisons being made, $k$. The Benjamini-Hochberg procedure [18], which we use in the main text, ranks all of the $p$ values in order from smallest to largest, and scales the Bonferroni corrected $p$ values by the rank $i: p \rightarrow p \times k / i$. This procedure provides greater statistical power when analyzing pairwise differences when one can treat the $k$ different comparisons as independent.

\section{Correlation analysis and multiple linear regression}

Correlation analysis is used to determine the strength of the relationship between two variables. Multiple linear regression is an extension of correlation analysis that allows one to look at the relationship between a dependent variable and multiple different independent variables (predictors). For example, the linear regression

$$
\mathrm{FMCE}=\beta_{0}+\beta_{1} \mathrm{AP}+\beta_{2} \mathrm{SAT}+\epsilon
$$

posits a linear relationship between FMCE score, AP physics score, and SAT score. Typically, one estimates the regression coefficients $\beta_{i}$ using the method of ordinary least squares, which minimizes the squared regression error $\epsilon^{2}$ with respect to the regression coefficients.

For valid ordinary least squares estimates of the regression coefficients, four conditions must be met [21],

(a) The observations are independent and drawn from the same distribution (i.e., the measure for each student is an independent observation).

(b) The error term is not correlated with AP score or SAT score and follows a standard normal distribution with constant variance.

(c) All variables have finite, nonzero, fourth moments.

(d) The independent variables (AP score and SAT score) are not linearly dependent, i.e., AP $\neq \gamma_{0}+\gamma_{1}$ SAT.

Conditions (a), (b) and (c) are met for this case, but condition (d) is a concern. The significance of the violation of condition (d), "multicollinearity," depends on the size of the correlation between the two variables in question. A strong correlation between variables leads to inflated estimates of the standard error of regression coefficients, making it difficult to disentangle the effects of the variables from one another. Typically, variance inflation is not considered to be a concern for correlations less than 0.9, but this will depend on the number of observations. Multicollinearity is considered to be a problem when the variance inflation factor [VIF $\left.=1 /\left(1-R^{2}\right)\right]$ is above 5 [21]. In our analysis it is only 1.5 . The variance inflation is under $50 \%$, and the qualitative results are not affected.
[1] Score distributions The College Board, 2018, retrieved from https://apscore.collegeboard.org/scores/about-ap -scores/score-distributions/.

[2] S. Kolluri, Advanced placement: The dual challenge of equal access and effectiveness, Rev. Educ. Res. 88, 671 (2018).

[3] D. Hestenes, M. Wells, and G. Swackhamer, Force concept inventory, Phys. Teach. 30, 141 (1992).

[4] R. Thornton and D. Sokoloff, Assessing student learning of Newton's laws: The force and motion conceptual evaluation and the evaluation of active learning laboratory and lecture curricula, Am. J. Phys. 66, 338 (1998).

[5] N.T. Belcher, Modeling instruction in AP Physics C: Mechanics and electricity and magnetism, Ph.D. thesis, University of South Carolina, 2017.

[6] L. E. Kost, S. J. Pollock, and N. D. Finkelstein, Characterizing the gender gap in introductory physics, Phys. Rev. ST Phys. Educ. Res. 5, 010101 (2009). 
[7] L. E. Kost-Smith, S. J. Pollock, and N. D. Finkelstein, Gender disparities in second-semester college physics: The incremental effects of a "smog of bias", Phys. Rev. ST Phys. Educ. Res. 6, 020112 (2010).

[8] Z. Hazari, R. H. Tai, and P. M. Sadler, Gender differences in introductory university physics performance: The influence of high school physics preparation and affective factors, Sci. Educ. 91, 847 (2007).

[9] P. M. Sadler and R. H. Tai, Success in introductory college physics: The role of high school preparation, Sci. Educ. 85, 111 (2001).

[10] R. H. Tai and P. M. Sadler, Gender differences in introductory undergraduate physics performance: University physics versus college physics in the USA, Int. J. Sci. Educ. 23, 1017 (2001).

[11] AP Physics C course description, The College Board, 2014, retrieved from https://apstudent.collegeboard.org/ apcourse/ap-physics-c-mechanics/course-details.

[12] H. D. Young and R. A. Freedman, University Physics with Modern Physics 14th ed. (Pearson, Boston, 2015).

[13] L. C. McDermott and P. S. Shaffer, Tutorials in Introductory Physics (Prentice- Hall, Upper Saddle River, NJ, 2002).

[14] Students reported a mix of SAT and ACT scores. We converted ACT scores to SAT scores using standard concordance tables provided by The College Board [15].

[15] Guide to the 2018 ACT/SAT concordance, The College Board, 2018, retrieved from https://collegereadiness .collegeboard.org/pdf/guide-2018-act-sat-concordance.pdf.

[16] Only 374 of the 431 students completed the post-test: 286 had taken an AP or college-level physics course, 51 had taken a high school physics course, and 37 had no prior physics courses. The FMCE prescores and math SAT scores were statistically indistinguishable for the two groups.

[17] G. V. Glass and K. D. Hopkins, Statistical Methods in Education and Psychology, 3rd ed. (Allyn \& Bacon, Needham Heights, MA, 1996).

[18] Y. Benjamini and Y. Hochberg, Controlling the false discovery rate: A practical and powerful approach to multiple testing, J. R. Stat. Soc. Ser. B 57, 289 (1995).

[19] E. L. Lehmann, Nonparametrics: Statistical Methods Based on Ranks (Springer, New York, 2006).

[20] S. Salehi, E. W. Burkholder, G. P. Lepage, S. J. Pollock, and C.E. Wieman, Demographic gaps or preparation gaps?: The large impact of incoming preparation on performance of students in introductory physics, Phys. Rev. Phys. Educ. Res. 15, 020114 (2019).

[21] J. H. Stock and M. W. Watson, Introduction to Econometrics, 3rd ed. (Pearson, Boston, 2015).

[22] S. Ramlo, Validity and reliability of the force and motion conceptual evaluation, Am. J. Phys. 76, 882 (2008).

[23] K. D. Mattern, B. F. Patterson, and J. L. Kobrin, The validity of SAT scores in predicting first-year Mathematics and English grades, 2012, retrieved from the Education Resources Information Center, https://files.eric.ed.gov/fulltext/ED 563105.pdf.

[24] P. Barniol and G. Zavala, Test of understanding of vectors: A reliable multiple-choice vector concept test, Phys. Rev. ST Phys. Educ. Res. 10, 010121 (2014).
[25] C. Dougherty, L. Mellor, and S. Jian, The Relationship between Advanced Placement and College Graduation (National Center for Educational Achievement, Austin, TX, 2006).

[26] S. Geiser and V. Santelices, The role of advanced placement and honors courses in college admissions, Research and Occasional Paper Series CSHE.4.04, 2004, retrieved from https://cshe.berkeley.edu/sites/default/files/publications/ rop.geiser.4.04.pdf.

[27] P. M. Sadler and G. Sonnert, High school advanced placement and success in college coursework in the sciences, in AP: A Critical Examination of the Advanced Placement Program, edited by P. M. Sadler, G. Sonnert, R. H. Tai, and K. Klopenstein (Harvard Education Press, Cambridge, MA, 2010), pp. 119-137.

[28] K. Klopfenstein and M. K. Thomas, Advanced placement participation: Evaluating the policies of states and colleges, in AP: A Critical Examination of the Advanced Placement Program (Harvard Education Press, Cambridge MA, 2010), (Ref. [26]), pp. 167-188, https://www.hepg.org/ hep-home/books/ap.

[29] A. Rodriguez (personal communication).

[30] Estimates of rates of nonparticipation as of 2000 were between $30 \%$ and $40 \%$, and had reached $50 \%$ by 2009 Ref. [31,32].

[31] National Research Council, Learning and Understanding: Improving Advanced Study of Mathematics and Science in U.S. High Schools (National Academies Press, Washington, DC, 2002).

[32] The High School Longitudinal Study of 2009 (HSLS:09), U.S. Department of Education, Institute of Education Sciences, National Center for Education Statistics.

[33] The 10th AP annual report to the nation, The College Board, 2014, retrieved from http://media.collegeboard .com/digitalServices/pdf/ap/rtn/10th-annual/10th-annual-apreport-to-the-nation-single-page.pdf.

[34] A. K. Nayak, Teaching of Physics (APH Publishing Corporation, New Delhi, 2007).

[35] L. Deslauriers and C. Wieman, Learning and retention of quantum concepts with different teaching methods, Phys. Rev. Phys. Educ. Res. 7, 010101 (2011).

[36] S. J. Pollock, Longitudinal study of student conceptual understanding in electricity and magnetism, Phys. Rev. ST Phys. Educ. Res. 5, 020110 (2009).

[37] R. M. Talbot, Taking an item-level approach to measuring change with the force and motion conceptual evaluation: An application of item response theory, School Sci. Math. 113, 356 (2013).

[38] R. Henderson, P. Miller, J. Stewart, A. Traxler, and R. Lindell, Item-level fairness in the force and motion conceptual evaluation and the conceptual survey of electricity and magnetism, Phys. Rev. Phys. Educ. Res. 14, 020103 (2018).

[39] J. Stewart, C. Zabriskie, S. DeVore, and G. Stewart, Multidimensional item response theory and the force concept inventory, Phys. Rev. Phys. Educ. Res. 14, 010137 (2018). 